DOI: 10.12957/demetra.2015.14412

\title{
Avaliação da padronização do porcionamento de uma unidade de alimentação e nutrição de um centro educacional infantil
}

\section{Evaluation of standarization portion in a foodservice unit in a children education center}

\author{
Marcela Barthichoto' \\ Andrea Carvalheiro Guerra Matias² \\ Mônica Glória Neumann Spinelli² \\ Edeli Simioni de Abreu² \\ 1 Universidade Federal de São Paulo. Programa \\ de Pós-graduação em Nutrição e Saúde da \\ Universidade Federal de São Paulo. São Paulo- \\ SP, Brasil. \\ ${ }^{2}$ Universidade Presbiteriana Mackenzie. Centro \\ de Ciências Biológicas e da Saúde, Cursos de \\ Nutrição e Tecnologia em Gastronomia. São \\ Paulo-SP, Brasil. \\ Correspondência / Correspondence \\ Andrea Carvalheiro Guerra Matias \\ E-mail:acgmatias@gmail.com
}

\section{Resumo}

A alimentação adequada é importante em qualquer fase da vida, mas na infância ela desempenha papel decisivo. Nessa fase ocorre um acelerado processo de crescimento e desenvolvimento, tanto físico como psicomotor, que depende de uma alimentação nutricionalmente equilibrada para que ocorram normalmente. Este estudo se propôs avaliar o padrão de porcionamento oferecido às crianças em uma Unidade de Alimentação e Nutrição de um centro educacional. Para avaliar se o porcionamento segue um padrão homogêneo em peso, as porções foram pesadas em quadruplicada, durante quatro dias. Verificou-se inadequação na padronização das porções nas diferentes faixas etárias, e na análise do cardápio verificou-se oferta adequada de macronutrientes e de fibras, e inadequada de micronutrientes. Conclui-se que há uma inadequação de padronização das porções, com consequente excesso de oferta e de restos alimentares.

Palavras-chave: Alimentação Escolar. Refeição. Serviços de Alimentação.

\section{Abstract}

At any stage of human life appropriate nutrition is important, but in childhood it plays a decisive role. At this stage there is an accelerated process of growth and development, both physical and psychomotor, which depends on a nutritionally balanced 
to occur normally. This study aimed to evaluate the pattern of portioning offered to children inan Educational Center Cafeteria. To assess whether the portioning follows a homogeneous weigh pattern, the portions were weighed quadrupled for four days. It was used digital accurately with up to $5 \mathrm{~kg}$ and an accuracy of $2 \mathrm{~g}$. There was inadequate standardization of parts among different age groups and analysis of the menu was found an adequate offer of macronutrients and fiber and inadequate micronutrients. It is concluded that there is inadequate serving standardization that takes to a oversupply and food remains.

Key-words: School Feeding. Meals. Food Services.

\section{Introdução}

Estudos sobre consumo alimentar de crianças brasileiras nas escolas são escassos, embora atualmente exista uma proporção relevante e crescente de crianças em idade pré-escolar e escolar, e tenha aumentado o número de escolas que oferecem refeições para crianças que estudam em período integral. Alguns estudos mostram que, embora de natureza multifatorial, os hábitos alimentares inadequados estão entre os fatores determinantes que mais repercutem desfavoravelmente sobre o estado nutricional das crianças. ${ }^{1.4}$

Embora as deficiências alimentares resultem em consequências importantes no desenvolvimento das crianças, a literatura que trata das questões da qualidade dos serviços de alimentação nas escolas é ainda escassa.

A qualidade em um serviço de alimentação é decorrente, entre outras coisas, da padronização dos processos Esta, além de auxiliar o preparo das refeições e favorecer a adequação da oferta energética, atua como fator de redução do desperdício alimentar, uma vez que o porcionamento padronizado e adequado evita o excesso de alimentos preparados e no prato, com consequente redução do descarte. Depende, ainda, da sensibilização em relação a esta questão e da capacitação dos trabalhadores em relação às quantidades ofertadas. ${ }^{5,6}$

Nesse contexto, o presente estudo se propôs a avaliar o padrão de porcionamento oferecido às crianças em uma Unidade de Alimentação e Nutrição (UAN) de um centro educacional. 


\section{Metodologia}

Trata-se de estudo de caráter transversal com coleta de dados primários, realizado nos meses de abril e maio de 2013, em uma UAN com gestão terceirizada, de um centro educacional localizado na zona sul no município de São Paulo.

Nesse centro educacional, as refeições são servidas pelas auxiliares de cozinha, sendo oferecidos dois padrões de porcionamento: o primeiro para os alunos categorizados como infantil (2 a 3 anos) e o segundo para os alunos categorizados como pré-escolares e escolares (4 a 10 anos). A avaliação do porcionamento foi realizada no período do jantar apenas para as crianças de 2-3 anos e de 7-10 anos, identificados neste trabalho como infantil e escolares, respectivamente. Segundo a administração da UAN, a padronização das porções é realizada segundo medidas caseiras preconizadas contratualmente entre o centro educacional e a terceirizada.

Com o intuito de avaliar se o porcionamento segue um padrão homogêneo em peso, as porções foram pesadas em quadruplicata, durante quatro dias. Para tanto, foi utilizada balança digital da marca Casíta, com capacidade para $5 \mathrm{~kg}$ e precisão de $2 \mathrm{~g}$.

Os dados coletados foram tabulados e apresentados por meio da distribuição das variáveis em número e porcentagem, bem como de medidas de tendência central e dispersão, com o auxílio do software Microsoft Office Excel®, versão 2010.

$\mathrm{O}$ estudo seguiu as diretrizes e normas que regulamentam as pesquisas envolvendo seres humanos, sendo aprovado eticamente através do número CIEP N011/04/13.

\section{Resultados}

No quadro 1 são apresentados os cardápios de quatro dias. 
Quadro 1. Preparações servidas no jantar pela Unidade de Alimentação e Nutrição de um centro educacional infantil, São Paulo-SP, 2013.

\begin{tabular}{|c|c|c|c|c|}
\hline $\begin{array}{l}\text { Composição } \\
\text { do cardápio }\end{array}$ & 10 dia & $2^{\mathbf{a}} \mathrm{dia}$ & $3^{\mathbf{a}}$ dia & $4^{\mathrm{a}} \mathrm{dia}$ \\
\hline $\begin{array}{c}\text { Prato } \\
\text { proteico }\end{array}$ & $\begin{array}{c}\text { Iscas de frango } \\
\text { aceboladas }\end{array}$ & $\begin{array}{l}\text { Picadinho de } \\
\text { carne }\end{array}$ & $\begin{array}{c}\text { Pescado ao } \\
\text { forno }\end{array}$ & Chester \\
\hline Guarnição & $\begin{array}{l}\text { Cuscuz de } \\
\text { legumes }\end{array}$ & - & Batata bolinha & $\begin{array}{c}\text { Mandioca } \\
\text { cozida }\end{array}$ \\
\hline Salada & Tomate & Alface & $\begin{array}{l}\text { Brócolis } \\
\text { refogado }\end{array}$ & Repolho \\
\hline Sobremesa & Ameixa & Abacaxi & Maçã & Laranja \\
\hline \multirow{2}{*}{ Prato base } & Arroz & \multirow{2}{*}{$\begin{array}{l}\text { Macarrão com } \\
\text { tomate cereja }\end{array}$} & Arroz & Arroz \\
\hline & Feijão & & Feijão & Feijão \\
\hline
\end{tabular}

Na tabela 1, são apresentados os valores do porcionamento das preparações servidas para as crianças do infantil.

Tabela 1. Distribuição da média $(n=4)$ de porcionamento em gramas das preparações servidas para as crianças do grupo infantil. São Paulo-SP, 2013.

\begin{tabular}{ccccccccc}
\hline Composição & $\begin{array}{c}\text { Dia 1 } \\
\text { Porção }(\mathrm{g}) \\
\text { do cardápio }\end{array}$ & $\begin{array}{c}\text { CV } \\
(\mathrm{X} \pm \mathrm{DP})\end{array}$ & $\begin{array}{c}\text { Dia } 2 \\
\text { Porção }(\mathrm{g}) \\
(\mathrm{X} \pm \mathrm{DP})\end{array}$ & $\begin{array}{c}\mathrm{CV} \\
(\%)\end{array}$ & $\begin{array}{c}\text { Dia } 3 \\
\text { Porção }(\mathrm{g}) \\
(\mathrm{X} \pm \mathrm{DP})\end{array}$ & $\begin{array}{c}\text { CV } \\
(\%)\end{array}$ & $\begin{array}{c}\text { Porção }(\mathrm{g}) \\
(\mathrm{X} \pm \mathrm{DP})\end{array}$ & $\begin{array}{c}\mathrm{CV} \\
(\%)\end{array}$ \\
\hline Arroz & $90,3 \pm 3,1$ & 3 & - & - & $71,8 \pm 10,8$ & 15 & $68,5 \pm 6,4$ & 9 \\
Feijão & $74,5 \pm 9,9$ & 13 & - & - & $57,5 \pm 14,3$ & 25 & $64,8 \pm 11,4$ & 18 \\
Macarrão & - & - & $85,5 \pm 5,8$ & 7 & - & - & - & - \\
Prato proteico & $30,0 \pm 4,5$ & 15 & $61,0 \pm 6,1$ & 10 & $36,5 \pm 8,4$ & 23 & $52,2 \pm 13,3$ & 25 \\
Guarnição & $56,3 \pm 8,6$ & 15 & - & - & $27,8 \pm 2,4$ & 9 & $8,5 \pm 1,3$ & 15 \\
Salada & $40,3 \pm 8,1$ & 20 & $11,0 \pm 0,2$ & 2 & $72,8 \pm 7,5$ & 10 & $17,2 \pm 4,3$ & 25 \\
Fruta & $24,3 \pm 7,3$ & 30 & $80,2 \pm 7,1$ & 9 & $33,5 \pm 7,4$ & 22 & $61,1 \pm 6,1$ & 10 \\
Peso total & 315,0 & & 237,7 & & 299,9 & & 273,3 & \\
\hline
\end{tabular}

a No dia 2 como prato base foi oferecido macarrão; DP- desvio padrão; X - média; CV - coeficiente de variação (DP/Xx100). 
Na tabela 2, são apresentados os dados do porcionamento das preparações servidas para as crianças do grupo pré-escolar e escolar.

Tabela 2. Distribuição da média $(n=4)$ de porcionamento das preparações servidas para as crianças do pré-escolar e escolar. São Paulo-SP, 2013.

\begin{tabular}{ccccccccc}
\hline $\begin{array}{c}\text { Composição } \\
\text { do cardápio }\end{array}$ & $\begin{array}{c}\text { Dia 1 } \\
\text { Porção }(\mathrm{g}) \\
(\mathrm{X} \pm \mathrm{DP})\end{array}$ & $\begin{array}{c}\text { CV } \\
(\%)\end{array}$ & $\begin{array}{c}\text { Dia } 2 \\
\text { Porção }(\mathrm{g}) \\
(\mathrm{X} \pm \mathrm{DP})\end{array}$ & $\begin{array}{c}\mathrm{CV} \\
(\%)\end{array}$ & $\begin{array}{c}\text { Dia } 3 \\
\text { Porção }(\mathrm{g}) \\
(\mathrm{X} \pm \mathrm{DP})\end{array}$ & $\begin{array}{c}\text { CV } \\
(\%)\end{array}$ & $\begin{array}{c}\text { Dia } 4 \\
\text { Porção }(\mathrm{g}) \\
(\mathrm{X} \pm \mathrm{DP})\end{array}$ & $\begin{array}{c}\text { CV } \\
(\%)\end{array}$ \\
\hline Arroz & $115,8 \pm 14,6$ & 13 & - & - & $124,5 \pm 10,6$ & 9 & $\begin{array}{c}123,8 \pm \\
38,3\end{array}$ & 31 \\
Feijão & $107,5 \pm 15,7$ & 15 & - & - & $98,0 \pm 16,1$ & 16 & $44,3 \pm 4,5$ & 10 \\
Macarrão & & & $208,8 \pm 5,4$ & 3 & & & & \\
Prato proteico & $76,8 \pm 4,3$ & 6 & $96,8 \pm 21,9$ & 23 & $56,5 \pm 17,3$ & 31 & $35,0 \pm 4,7$ & 13 \\
Guarnição & $87,3 \pm 12,1$ & 14 & & 3 & $42,8 \pm 6,9$ & 16 & $81,8 \pm 4,6$ & 6 \\
Salada & $66,3 \pm 11,3$ & 17 & $29,5 \pm 1$ & 3 & $76,3 \pm 10,8$ & 14 & $19,3 \pm 4,1$ & 21 \\
Fruta & $40,8 \pm 7,8$ & 19 & $101,8 \pm 10,3$ & 10 & $68,0 \pm 7,2$ & 11 & $163,8 \pm 9,2$ & 6 \\
\hline Peso total & 494,5 & & 436,9 & & 466,1 & & 468 & \\
\hline
\end{tabular}

a No dia 2 como prato base foi oferecido macarrão. DP- desvio padrão; X - média; CV - coeficiente de variação (DP/Xx100).

Observou-se, para ambos os grupos, uniformidade no peso total médio da refeição ao longo da semana, com variação de 238 a $315 \mathrm{~g}(\mathrm{CV}=12 \%)$ para o infantil, e437 a $495 \mathrm{~g}(\mathrm{CV}=5 \%)$ para o pré-escolares e escolares.

\section{Discussão}

A Estratégia Global, criada pela Organização Mundial da Saúde, ${ }^{7}$ reconhece que a escola é um espaço privilegiado para a promoção da saúde e desempenha papel fundamental na formação de valores, hábitos e estilos de vida, entre eles o da alimentação.

Avaliando o cardápio oferecido (quadro 1), verifica-se que os mesmos estão qualitativamente balanceados, sendo compostos por verduras, legumes, frutas, carnes magras e sem a presença de doces como sobremesa. No entanto, o mesmo poderia estar mais bem equilibrado no quesito “combinação das cores das preparações". Recomenda-se variar o tipo de frutas, legumes e verduras 
consumidos na semana. Para a elaboração do cardápio, deve-se procurar combinar verduras e legumes de maneira que o prato fique colorido, garantindo assim diferentes nutrientes e atratividade. ${ }^{8}$

A escola da presente pesquisa oferece diariamente hortaliças e frutas de forma a estimular o hábito alimentar adequado (quadro 1). Estudo brasileiro refere baixo consumo de frutas, verduras e legumes por crianças, sendo importante criar ações que revertam este cenário, uma vez que esses alimentos são essenciais para o desenvolvimento infantil por serem fontes de vitaminas, minerais e fibras alimentares. ${ }^{9}$ Além disso, a ingestão de frutas, legumes e verduras é um fator protetor contra o desenvolvimento da obesidade, devido à menor densidade energética desses alimentos e à sua capacidade de gerar sensação de saciedade, em decorrência da atuação das fibras alimentares. As fibras também têm ação hipocolesterolêmica e, quando associadas ao consumo de água, previnem a constipação. ${ }^{8}$

O cardápio desta escola não oferece preparações ricas em açúcar, contrariando outros estudos, como o de Zveibrücker \& Miraglia ${ }^{10}$ e Barbosa et al.,, ${ }^{11}$ que encontraram alto consumo deste ingrediente. $\mathrm{O}$ açúcar possui índice glicêmico elevado, contribuindo para o aumento da densidade energética da dieta sem proporcionar um valor nutritivo adequado $\mathrm{O}$ alto consumo por crianças, além de ser prejudicial para o estado nutricional, é fator de risco para o desenvolvimento de doenças crônicas não transmissíveis (DCNT), tal como diabetes, além de predispor ao aparecimento de cáries dentárias. ${ }^{12}$

No que concerne ao tamanho da porção, o Guia Alimentar para a População Brasileira, de 2006, estimava $117 \mathrm{~g}$ de arroz polido cozido. ${ }^{13}$ Neste estudo, a porção para o grupo infantil variou de 69 a $90 \mathrm{~g}$. O tamanho das porções deve ser menor quando comparado com as quantidades para o adulto no tocante à maioria dos grupos dos alimentos, mantendo-se inalterado apenas para o grupo das frutas, leites, queijo e iogurte. Tal redução é importante, pois nessa fase ocorre grande variação em relação ao apetite, bem como limitada capacidade gástrica das crianças nessa faixa etária. ${ }^{14}$

Já a idade escolar é caracterizada por um período de crescimento com exigências nutricionais maiores, devido ao trato gastrintestinal estar mais desenvolvido, e seu volume gástrico ser compatível ao de um adulto. ${ }^{15}$

Com relação à variação no porcionamento (observada pelo coeficiente de variação), alguns resultados estão dentro do esperado, como no caso das frutas, em que se observou alta ou baixa variação, conforme a apresentação inteira ou fracionada. No grupo infantil, o peso da ameixa e maçã inteiras e do abacaxi picado apresentaram variação de 30\%, 22\% e 9\%, respectivamente.

Quanto ao porcionamento do prato proteico, foram notadas discrepâncias, o que não poderia ocorrer, uma vez que a UAN, sendo terceirizada, tem o peso da porções previsto contratualmente. Nas refeições do grupo infantil (tabela 1), a porção média de picadinho de carne (dia 2) correspondeu ao dobro da preparação iscas de frango (dia 1), 61g e 30g, respectivamente. Para os pré-escolares e 
escolares, essa diferença ocorreu entre as preparações iscas de frango aceboladas (dia 1) e chester (dia 4), ambos com ave, com média de 77 e 35g, respectivamente. Destaque para o menor porcionamento da preparação chester (dia 4) para as crianças do grupo pré-escolar e escolares em relação do grupo infantil, 33\% menor, com média de $35 \mathrm{~g}$ e $52 \mathrm{~g}$, respectivamente (tabelas 1 e 2).

As diferentes gramagens do peso médio diário dos pratos proteicos indicam desigualdade na distribuição dos macronutrientes do cardápio, uma vez que as porções variaram em até $100 \%$. Para o grupo infantil, no dia 1 foram oferecidas porções médias de $30 \mathrm{~g}$ de iscas de frango aceboladas, e $60 \mathrm{~g}$ de picadinho de carne no dia 2. Os escolares por sua vez, receberam $35 \mathrm{~g}$ de chester, no dia 4, e $77 \mathrm{~g}$ de iscas de frango aceboladas no dial.

Esta variabilidade do tamanho das porções com relação aos aspectos administrativos da UAN impacta nos custos, sobretudo quando se refere ao alimento proteico, uma vez que este responde pelo maior custo alimentar. Possivelmente, todas estas variações sugerem problemas na capacitação dos manipuladores e/ou na padronização dos utensílios de servir. ${ }^{5}$

De acordo com Almeida \& Fernandes, ${ }^{16}$ para o planejamento do cardápio, três considerações devem ser levantadas: valor energético, definição do número de porções e distribuição das porções no decorrer das refeições.

O índice de resto é um indicativo de aceitação, desperdício e excesso de oferta. ${ }^{5}$ Em refeitórios escolares, alguns fatores contribuem significativamente para o desperdício, como a dificuldade de adequar o porcionamento para as diferentes faixas etárias, o pequeno intervalo entre as refeições e o fato de o porcionamento, na maioria das vezes, ser feito por adultos. ${ }^{17-19}$ Segundo Holland, ${ }^{20}$ a distribuição das refeições pelo sistema de autosserviço pode ser implantada a partir do módulo maternal, permitindo que a criança se sirva da quantidade que ela realmente quer comer e reduzindo, assim, o desperdício.

O quadro 2 apresenta outros estudos que avaliaram porcionamento para escolares.

Quadro 2. Quadro comparativo entre os valores mínimos e máximos de porcionamento do estudo atual com outros trabalhos, São Paulo-SP, 2013

\begin{tabular}{|c|c|c|c|c|c|}
\hline \multirow{2}{*}{} & \multirow{2}{*}{ Infantil } & \multirow{2}{*}{ Escolares } & \multicolumn{3}{|c|}{ Outros estudos } \\
\cline { 4 - 6 } & & & Pioltine e Spinelli ${ }^{19}$ & Faquim et al. $^{1}$ \\
\hline Idade (anos) & 2 a 3 & 7 a 10 & 2 a 5 & 6 a 10 & 5 a 6 \\
\hline Arroz $(\mathrm{g})$ & $69-90$ & $116-209$ & $33-46^{\text {a }}$ & 77 & $30-55$ \\
\hline Feijão $(\mathrm{g})$ & $58-75$ & $44-108$ & 28 & 86 & 80 \\
\hline Prato proteico $(\mathrm{g})$ & $30-61$ & $35-97$ & 27,5 & 58 & $35-60$ \\
\hline
\end{tabular}

a soma do arroz branco e integral. 
Sugere-se, para o presente estudo, um excesso de oferta de alimentos em peso, independentemente da faixa etária das crianças. A tabela 1 mostra para a faixa etária de 2-3 anos (grupo infantil), idade de desaceleração do crescimento, com consequente redução de consumo, ${ }^{18}$ uma oferta de 238 a 315g. Faquim, Oliveira e Spinelli ${ }^{1}$ referem para a idade de 5-6 anos, uma oferta de 228$315 \mathrm{~g}$ (sem sobremesa), e Pioltine \& Spinelli, ${ }^{21}$ oferta de 193 a $270 \mathrm{~g}$ para crianças de $2-5$ anos. No primeiro estudo, o consumo médio foi de 121 a $144 \mathrm{~g}$, com resto alimentar entre 22,6 a 47,6\% do total oferecido. No segundo, o consumo médio foi $204 \mathrm{~g}$, ou seja, $13 \%$ de resto alimentar. Para os escolares de 7-10 anos, observou-se oferta média de 437 a 495g. Pioltine \& Spinelli ${ }^{21}$ referem para crianças de 6-10 anos uma oferta que variou de 418 a $564 \mathrm{~g}$, com consumo médio de $409 \mathrm{~g}$, e resto alimentar de 19,4\%. Outros estudos, como Campos, Viana \& Rocha, ${ }^{22}$ e Pikelaizen \& Spinelli, ${ }^{17}$ também observaram elevado índice de resto alimentar, $31 \%$ e 21\%, respectivamente.

Outro agravante é que o excesso de oferta pode ter como consequência o excesso de consumo. Segundo Disantis et al., ${ }^{23}$ a utilização de pratos de tamanho normal para adultos leva a um porcionamento maior e, consequentemente, ao aumento de consumo.

\section{Conclusão}

Conclui-se que há inadequação de padronização das porções, com consequente excesso de oferta e de restos alimentares.

São necessários mais estudos que investiguem o real consumo, uma vez que o excesso de oferta pode predispor à obesidade, e um alto índice de restos pode repercutir negativamente na saúde financeira da empresa e sustentabilidade ambiental.

\section{Referências}

1. Faquim NB, Oliveira T, Spinelli MGN. Porcionamento, consumo e desperdício em um restaurante escolar. Revista UNIVAP 2012; 18:71-77.

2. Castro TG, Campos FM, Priore SE, Coelho FMG, Campos MTFS, Franceschini SCC, Rangel AA. Saúde e nutrição de crianças de 0 a 60 meses de um assentamento de reforma agrária, Vale do Rio Doce, MG, Brasil. Revista de Nutrição 2004; 17(2):167-76

3. Fisberg RM, Bueno MB, Marchioni DML. Evolução nutricional de crianças atendidas em creches públicas no município de São Paulo, Brasil. Rev. Panam. Salud Pública 2003; 14:165-70. 
4. Silva DG, Franceschini SCC, Priore SE, Euclydes MP, Araújo RMA, Ribeiro SMR, et al. Anemia ferropriva em crianças de 6 a 12 meses atendidas na rede pública de saúde do município de Viçosa, Minas Gerais. Revista de Nutrição 2002; 15(3):301-8.

5. Abreu ES, Spinelli MGN, Pinto AMS. Gestão de Unidades de Alimentação e Nutrição: um modo de fazer. v. 1. 5a ed. São Paulo: Metha; 2013.

6. Freire P. Educação e mudança. 24 ed. Rio de Janeiro: Paz e Terra; 2001.

7. World Health Organization. Global strategy on diet, physical activity and health. In: Fifty-Seventh World Health Assembly [Internet] 2004. Acesso em: 30 nov. 2013. Disponível em: http://apps.who. int/gb/ebwha/pdf_files/WHA57/A57_R17-en.pdf

8. Brasil. Ministério da Saúde. Guia alimentar para a população brasileira: promovendo a alimentação saudável. Brasília: Ministério da Saúde; 2006.

9. Menegazzo M, Fracalossi K, Fernandes AC, Medeiros NI. Avaliação qualitativa das preparações do cardápio de centros de educação infantil. Revista de Nutrição 2011; 24(2):243-251.

10. Zveibrücker FP, Miraglia FM. Avaliação do consumo alimentar de pré-escolares frequentadores de EMEIs no Município de Nova Santa Rita, RS. CIPPUS - Revista de Iniciação Científica do Unilasalle 2012;1(1):63-77.

11. Barbosa RMS, Croccia C; Carvalho CGN, Franco VC, Salles-Costa R, Soares EA. Consumo alimentar de crianças com base na pirâmide alimentar infantil. Revista de Nutrição 2005; 18(5):633-641.

12. Menegazzo, M; Fracalossi, K; Fernandes, Medeiros AC, Neiva, I. Avaliação qualitativa das preparações do cardápio de centros de educação infantil. Revista de Nutrição 2011; 24(2):243-251.

13. Brasil. Ministério da Saúde. Guia alimentar para a população brasileira: promovendo a alimentação saudável. Brasília: Ministério da Saúde; 2006.

14. Philippi ST, Cruz ATR, Colucci ACA. Pirâmide alimentar para crianças de 2 a 3 anos. Revista de Nutrição 2003; 16(1):5-19.

15. Fagiole D. Educação nutricional na infância e adolescência: planejamento, intervenções, avaliação e dinâmicas. São Paulo: RCN; 2008.

16. Almeida CAN, Fernandes GC. A importância do porcionamento na alimentação balanceada. Inter. J. Nutr. 2011; 4(3):53-59.

17. Pikelaizen C, Spinelli MGN. Avaliação do desperdício de alimentos na distribuição do almoço servido para estudantes de um colégio privado em São Paulo, SP. Revista UNIVAP 2013; 19(33):5-12.

18. Vitolo MR. Da gestação ao envelhecimento. Rio de Janeiro: Rubio; 2012.

19. Spinelli MGN, Franciozi TM. Porcionamento e desperdício em unidade de alimentação escolar. Nutrição em Pauta 2013; 118:1-7.

20. Holland CV. A creche e seu papel na formação de práticas alimentares [dissertação]. São Paulo: Faculdade de Saúde Pública, Universidade de São Paulo; 1999. 
21. Pioltine MB; Spinelli MGN. Análise da oferta alimentar em uma escola privada do município de São Paulo. Revista UNIVAP 2012; 31(18):48-57

22. Campos V, Viana I, Rocha A. Estudo de desperdícios alimentares em meio escolar. Nutrição em Pauta 2011; 19(109): 60-64. 2011

23. Disantis KI, Birch LL, Davey A, Serrano EL, Zhang J, Bruton Y, Fisher J. Plate size and children's appetite: effects of larger dishware on self-served portions and intake. Pediatrics 2013; 131(5):1451-58.

Recebido: 30/1/2015

Revisado: $10 / 3 / 2015$

Aprovado: 05/5/2015 